\title{
KARE - A Patent Protected AI based Technology into Hospital and Healthcare
}

\author{
G.R.Vaisshalli ${ }^{1}$, Apar Gupta ${ }^{2 *}$, Nishu Agarwal ${ }^{3}$, Abhishek Ghosh ${ }^{4} \&$ Muskan Sharma ${ }^{5}$ \\ ${ }^{1-5}$ Blu Ocean Studios Private Limited, India. \\ A company registered under the companies Act 2013, DPIIT recognized.
}

Copyright: (C) 2021 Apar Gupta et al. This is an open access article distributed under the terms of the Creative Commons Attribution License, which permits unrestricted use, distribution, and reproduction in any medium, provided the original author and source are credited.

\section{ABSTRACT}

Artificial Intelligence (AI) is a technology that, when linked with healthcare apps and smart wearable devices, can anticipate the onset of health issues in users by gathering and analyzing their health data. The integration of AI with smart wearable devices offers a wide range of potential applications in smart healthcare, however there is an issue with the black box operation of AI models' judgments, which has led in a lack of accountability and trust in the decisions made. In the field of healthcare, transparency, outcome tracing, and model improvement are all important. Healthcare providers can be more watchful and proactive in their interactions with patients thanks to the Internet of Things. Wheelchairs, defibrillators, nebulizers, oxygen pumps, and other monitoring devices are all tracked in real time utilizing IoT devices with sensors.

Keywords: Artificial Intelligence, Healthcare, Internet of Things, Blu Ocean Studios Private Limited, Intellectual Property, Kare ${ }^{\mathrm{TM}}$

\section{Introduction}

Patients' interactions with doctors were limited before the Internet of Things to visits, teleconferences, and text messages. There was no means for doctors or hospitals to regularly assess patients' health and make appropriate recommendations. Remote monitoring in the healthcare industry is now possible thanks to Internet of Things (IoT)-enabled gadgets, which have the ability to keep patients safe and healthy while also empowering clinicians to provide superior treatment. Patients' participation and happiness have increased as interaction with doctors has become easier and more efficient. Furthermore, remote monitoring of a patient's health helps to shorten hospital stays and avoid re-admissions. One field where IoT and AI are having a huge impact is healthcare, which is constantly under pressure to reduce costs while serving a rapidly growing ill population. These technologies can help healthcare firms reap the benefits of an increasingly networked and responsive world. Patients, physicians, payers, and drug developers would all benefit if the industry could accomplish more interconnection within a cohesive ecosystem. The Internet of Things (IoT) and artificial intelligence (AI) are revolutionizing the way we diagnose and treat illnesses. They can speed up the diagnostic process and help avoid mistakes by using both historical data and patient information. AI technology like neural networks can quickly examine the large quantity of information available to clinicians. It's a two-way street: IoT and AI require each other. The Internet of Things necessitates the processing of massive amounts of data that must be deciphered and put to use. As a result, AI algorithms may and should be used to enhance IoT-related tasks in order to provide really meaningful experiences for users and/or consumers. Because the Internet of Things is a relatively new technology that links billions of smart gadgets, it is not without flaws. Such criteria as IoT data transfer accuracy and speed, for example, have yet to be addressed. Furthermore, an artificial intelligence system not only imitates the way humans execute things, but it also learns from the patterns it creates. AI relies heavily on this self-improvement mechanism. In general, Artificial Intelligence has a lot to offer the Internet of Things. The Internet of Things (IoT) powered by AI has the potential to play a big role in smart healthcare by providing better insight into healthcare data and enabling personalized care. 


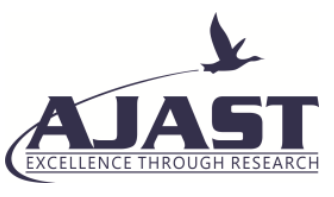

Asian Journal of Applied Science and Technology (AJAST)

Volume 5, Issue 4, Pages 51-54, Oct-Dec 2021

In the restricted sense, it refers to AI software that is embedded in IoT devices and works in conjunction with fog or edge computing solutions to provide intelligence to IoT devices. The essential phases for the smart and efficient use of AI algorithms in IoT devices are tracking (gathering), monitoring (analyzing), control, optimization (training), and automation (modelling, forecasting). When they operate together, they can alleviate the administrative strain on clinical personnel. With better clinical processes, medical officers will be able to spend more time with patients, resulting in a more patient-centric approach to healthcare service delivery. To be more precise, Data from wearable, ingestible, and embedded sensors, as well as mobility and device usage patterns, can be successfully collected and processed to expose crucial situations using state-of-the-art Artificial Intelligence and Machine Learning (ML/DL) based methodologies. The main uses of AI enabled IOT are: Medical personnel, patients, and inventory management, Management of chronic diseases, Drug administration, Time spent in the emergency department is reduced, Health monitoring from faraway. Besides this, Intelligent Automation which is the fusion of Robotic Process Automation, Artificial Intelligence and Machine learning is a new trend to keep an eye on! Digital workers (Intelligent Automation) process at machine speed and eliminates errors, allowing human workers to focus on higher-value jobs and deliver better direct patient care. Healthcare payers can benefit from intelligent automation in a variety of ways. However, identifying significant operations that can be automated utilizing Artificial Intelligence, Robotic Process Automation is critical.

\subsection{AI and IoT Overview}
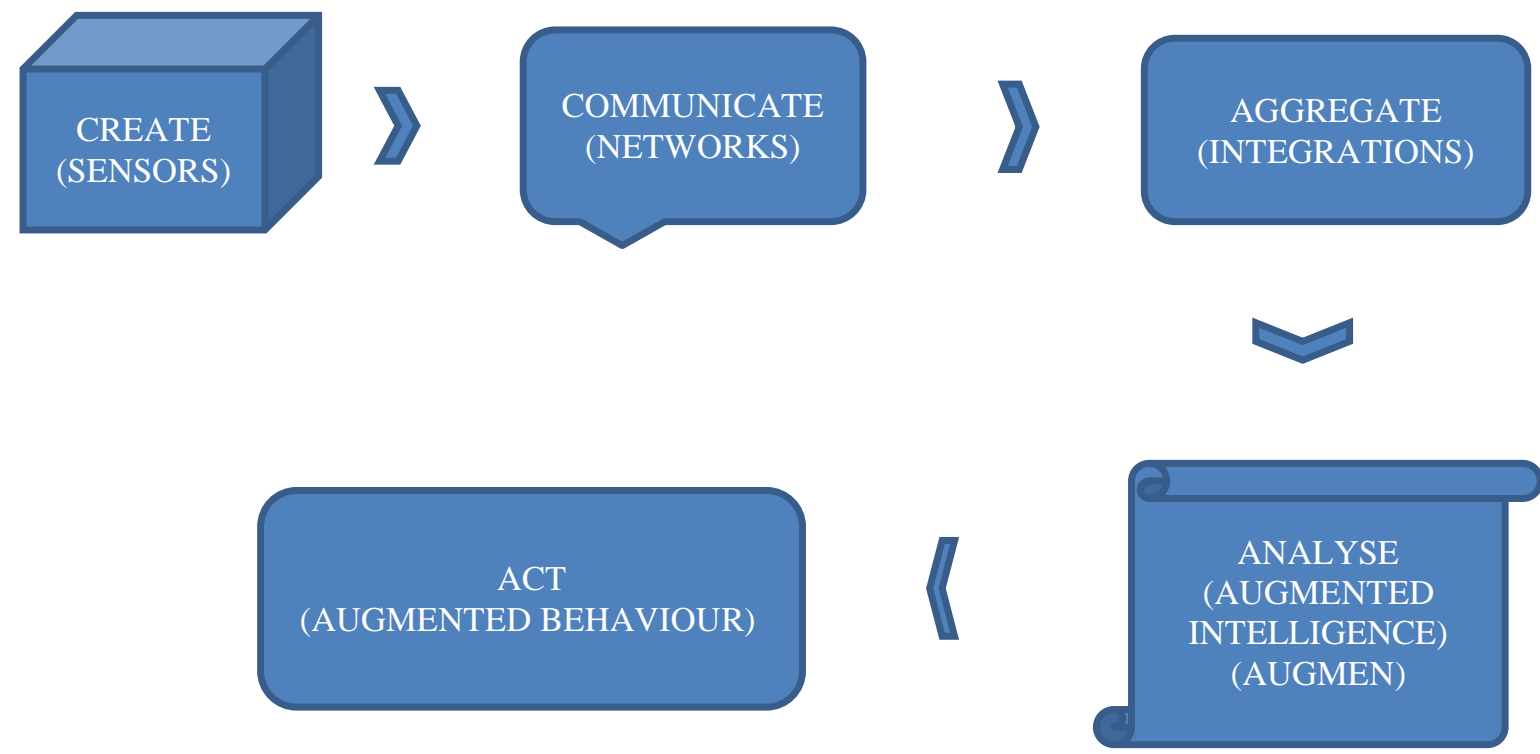

\section{Key Aspects of Digital Business Model}

\subsection{Remote ICU monitoring systems}

E-ICU arrange interfaces nearly all of its ICUs in such a way that a group of specialists can always screen basic patients in inaccessible offices and suggest treatment choices after investigating the therapeutic history and treatment alternatives for patients battling for their lives in genuine time after checking on therapeutic history and patients battling for their lives in genuine time. 


\subsection{RFID-IoT}

Employing a Fingerprint-IoT-based persistent administration apparatus, few hospitals oversee normal bottlenecks and robotize the utilize of diverse offices by moving around patients productively over an arrangement of rehashed demonstrative strategies.

\subsection{Unique Hospital Identification (UHID)}

UHID guarantees patients in India have a secure electronic record, which can moreover, be built into wearable restorative gadgets. UHID, combined with IoT, significantly speeds up determination and treatment as doctors can presently get to radiology and CT check reports through tablets or smartphones anyplace, and give recommendations to the care group.

\subsection{Leverage Big Data and Predictive Analytics}

Many hospitals have presently had advanced instruments to induce patients to see and to utilize huge information to improve client benefit and goad advancement through prescient investigation. When serving non-safety and latency sensitive IoT applications, traditional cloud-based architectures for Big Data Analysis can provide good performance and dependability.

\subsection{Telemedicine}

Hospitals have set up health-care centers in inaccessible zones by setting up a center, and connection telemedicine demonstrates that permits expansive masters in cities to treat patients over the web at far-off zones. IoT-blended AI applications can be used to predict liable epidemic outbreaks based on the past data, environmental, regional, and geographic data for better prevention and improved public health (Kankanhali, Charalabidis, \& Mellouli, 2019).

There is also a new term called Internet of Medical Things (IoMT), which is used to describe the technology in the medical field which are connected. This IoMT sector is growing in a much faster way and is predicted to reach US $\$ 158$ billion by the year 2022 (Dash, 2020). But, the recent pandemic will make the growth slower. Some of the widely classified categories in this IoMT segment are In-patient surveillance, imaging, telemedicine, and remote consultancy, healthcare involving wearable, management and workflow management.

Digital pathology involving the identification of cancer and cardio-vascular disease can be made possible by the application of AI (Artificial Intelligence) and ML (Machine Learning) which has caused a greater impact in the adaptive software development. Z-wave or Zig-Bee is used for the communicating and also used to gather and send collected information to the nursing stations about the patients (Kankanhali, Charalabidis, \& Mellouli, 2019). Collecting and managing data in healthcare is an important aspect and Big data and Artificial Intelligence (AI) helps to sort this process in easier way and also helps to predict and analyze any future outbreaks or failures.

The processing capacity required to analyse and make sense of the huge volumes of data generated by IoT devices is provided by artificial intelligence (AI) or machine learning algorithms. In AI - powered IoT sensors, AI algorithms are used which allows data processing to take place on the device itself, rather than relying on third party services like cloud computing. The device provides greater predictive insights in terms of requirements. 


\section{Conclusion}

The major goal of health-related AI applications is to investigate the links between preventative or treatment methods and patient outcomes. Diagnoses, treatment protocol creation, drug research, personalized medicine, and patient monitoring and care are all areas where AI systems are being used.

\section{Future Scope}

Within an IoT device, AI processing necessitates innovative solutions that fulfill the power, speed, and size requirements. To do this, the trend is moving towards machine learning-optimized integrated circuits. Edge AI refers to this style of processing. By computing locally, edge AI could aid in real time engagement with patients. It will also save time when compared to cloud-based computing, which is crucial when dealing with patients who have major health issues. IoT is set to become a vital element of healthcare in the United States and around the world as technologies mature and improve. Here are a few of the problems preventing IoT adoption in the healthcare industry.

\section{Declarations}

\section{Source of Funding}

This research did not receive any grant from funding agencies in the public, commercial, or not-for-profit sectors.

\section{Competing Interests Statement}

The authors declare no competing financial, professional and personal interests.

\section{Consent for publication}

Authors declare that they consented for the publication of this research work.

\section{References}

[1] Pawar, U., O’Shea, D., Rea, S., \& O’Reilly, R., (2020). Explainable AI in Healthcare. 2020 International Conference on Cyber Situational Awareness, Data Analytics and Assessment (CyberSA), pp.1-2, doi: 10.1109/ CyberSA49311.2020.9139655.

[2] Mohanta, B., Das, P., \& Patnaik, S., (2019). Healthcare 5.0: A Paradigm Shift in Digital Healthcare System Using Artificial Intelligence, IOT and 5G Communication. 2019 International Conference on Applied Machine Learning (ICAML), pp.191-196, doi: 10.1109/ICAML48257.2019.00044.

[3] Thota, C., Sundarasekar, R., Manogaran, G., Varatharajan, R., \& Priyan, M.K., (2018). Centralized fog computing security platform for IoT and cloud in the healthcare system. In Fog Computing: Breakthroughs in Research and Practice. IGI global, pp.365-378.

[4] Amin, R., Kumar, N., Biswas, G.P., Iqbal, R., \& Chang, V., (2018). A lightweight authentication protocol for IoT-enabled devices in distributed Cloud Computing environment. Future Gen. Comput. Syst. 78: 1005-1019. 